\title{
Lungenembolie: Subkutane Behandlung mit niedermolekularem Heparin?
}

\author{
Pulmonary Embolism: Subcutaneous Treatment with \\ Low-Molecular-Weight Heparin?
}

Die akute mechanische Verlegung der pulmonalen Strombahn mit thrombotischem Material ist in mehr als 90\% der Fälle eine Folge einer Thrombose im Bereich der tiefen Beinvenen, wesentlich seltener im Bereich des rechten Herzens oder der oberen Extremitäten. Das klinische Spektrum ist breit und reicht von asymptomatischen Verläufen bis zu fulminanten, lebensbedrohlichen Verläufen mit akutem Cor pulmonale, Schock und schwerer arterieller Hypoxie. Gegenwärtige Therapiemaßnahmen beinhalten bei submassiven Lungenembolien eine therapeutische Heparinisierung mit intravenös verabreichtem, unfraktioniertem Heparin mit einer Verlängerung der partiellen Thromboplastinzeit auf das 2,5- bis 3fache der Norm mit anschließender überlappender oraler Antikoagulation mit Cumarinen. Weiterhin kommen - insbesondere bei fulminanten Verläufen - die thrombolytische Therapie und - als ultima ratio - die chirurgische oder katheterbasierte Embolektomie zur Anwendung.

Ergebnisse von klinischen Studien der jüngeren Vergangenheit haben gezeigt, dass die subkutane Verabreichung von niedermolekularem Heparin ein sicheres und effektives Therapieregime bei der Behandlung tiefer Beinvenenthrombosen ist [1 - 4]. In einer von Gould 1999 veröffentlichten Metaanalyse konnte gezeigt werden, dass bei Patienten mit tiefer Beinvenenthrombose die Behandlung mit niedermolekularem Heparin - im Vergleich zu intravenös verabreichtem, unfraktioniertem Heparin - weniger Blutungskomplikationen hervorruft, seltener $\mathrm{zu}$ erneuten thromboembolische Ereignissen führt und die Mortalität signifikant reduziert [1]. Weniger klar ist die Rolle von niedermolekularem Heparin bei der Behandlung von akuten Lungenembolien, und die klinische Praxis besteht weiterhin darin, solche Patienten mit unfraktioniertem Heparin intravenös zu behandeln.
Ziel einer kürzlich publizierten Metaanalyse von D. J. Quinlan u. Mitarb. war deshalb der Vergleich zwischen niedermolekularem, subkutan verabreichtem Heparin und intravenös verabreichtem, unfraktioniertem Heparin hinsichtlich Sicherheit und Effektivität bei der Behandlung von Lungenembolien [5].

Es handelt sich um eine Metaanalyse von 12 randomisierten, kontrollierten Studien und schließt insgesamt 1951 Patienten mit submassiver Lungenembolie oder asymptomatischer Lungenembolie bei symptomatischer tiefer Beinvenenthrombose ein. Grundlage zur Auswahl der Studien waren die Literaturdatenbanken Medline, Embase und Cochrane Library.

Primärer Endpunkt war das erneute Auftreten von einem thromboembolischen Ereignis (tiefe Beinvenenthrombose, Lungenembolie) während des Behandlungszeitraums, sekundäre Endpunkte waren das erneute Auftreten von einem thromboembolischen Ereignis innerhalb von 3 Monaten, die Mortalität und das Auftreten von Blutungskomplikationen.

In der Gruppe der mit niedermolekularem Heparin behandelten Patienten wurde eine niedrigere Häufigkeit erneuter thromboembolischer Ereignisse während des Behandlungszeitraums (1,4 vs. 2,4\% in der Gruppe der mit unfraktioniertem Heparin behandelten Patienten) und innerhalb von 3 Monaten (3,0 vs. 4,4\%) beobachtet. Diese Unterschiede waren jedoch nicht statistisch signifikant. Ebenso fanden sich keine signifikanten Unterschiede hinsichtlich Mortalität am Ende des Behandlungszeitraumes (1,4 vs. 1,2\%) und nach 3 Monaten (4,7 vs. 6,1\%). Größere Blutungskomplikationen traten in $1,4 \%$ der mit niedermolekularem Heparin behandelten Patienten auf. Im Vergleich hierzu erlitten 
2,3\% der mit unfraktioniertem Heparin behandelten Patienten größere Blutungen. Kleinere Blutungskomplikationen traten häufiger bei niedermolekularem Heparin auf (6,8 vs. 5,5\%). Auch diese Unterschiede waren nicht statistisch signifikant.

Für Patienten mit nicht-fulminanter Lungenembolie besteht ein zunehmendes Interesse, niedermolekulares, subkutan applizierbares Heparin anstelle von unfraktioniertem, intravenös zu verabreichendem Heparin therapeutisch einzusetzen. Unfraktioniertes Heparin hemmt Faktor Xa und Thrombin gleichermaßen, während niedermolekulares Heparin präferenziell Faktor Xa inhibiert. Wesentliche Argumente für den Einsatz von niedermolekularem, subkutan applizierbarem Heparin bestehen in einer Erhöhung der Lebensqualität des Patienten und einer potenziellen Kostenreduktion im Vergleich zu unfraktioniertem Heparin, insbesondere weil die subkutane Applikation eine Behandlung zu Hause, d.h. außerhalb des stationären Rahmens, ermöglicht [3,4]. In einer Studie aus dem Jahre 1999 konnte gezeigt werden, dass eine Behandlung von Patienten mit tiefer Beinvenenthrombose mit niedermolekularem Heparin im Vergleich zu einer intravenösen Behandlung mit unfraktioniertem Heparin vor allem dann kostengünstiger ist, wenn $8 \%$ dieser Patienten vollständig ambulant behandelt bzw. wenn 13\% dieser Patienten frühzeitig aus dem Krankenhaus entlassen werden können [6]. Weiterhin wird niedermolekulares Heparin in einer gewichtsadaptierten, festen Dosierung verabreicht und bedarf keiner Kontrolle von Blutgerinnungsparametern.

Neben diesen Vorteilen hinsichtlich Kosten und Einfachheit der Anwendung hat sich niedermolekulares, subkutan verabreichtes Heparin auch hinsichtlich Sicherheit und Effektivität in zahlreichen Studien als mindestens gleichwertig im Vergleich zu unfraktioniertem, intravenös verabreichtem Heparin in der Behandlung tiefer Beinvenenthrombosen erwiesen [1-4]. Außerdem ist die Behandlung mit niedermolekularem Heparin im Vergleich zur Behandlung mit unfraktioniertem Heparin in einem deutlich geringerem Prozentsatz mit der Entwicklung einer Heparin-induzierten Thrombopenie assoziiert [7].

In der vorliegenden Studie von D. J. Quinlan u. Mitarb. zeigte sich kein signifikanter Unterschied hinsichtlich des Auftretens von erneuten thromboembolischen Ereignissen, Mortalität und Blutungskomplikationen zwischen beiden Behandlungsformen. Ein wesentlicher Nachteil der vorliegenden Studie besteht jedoch in der für eine solche Meta-Analyse doch relativ geringen Anzahl von Patienten in Verbindung mit einer insgesamt eher niedrigen Frequenz der den primären oder sekundären Parametern zugrunde liegenden Ereignissen (Tod, erneutes thromboembolisches Ereignis, Blutung), die letztlich keine verlässliche Aussage zulassen, ob es signifikante Unterschiede zwischen beiden Therapieregimen gibt. Weiterhin liefert die vorliegende Studie keine Ergebnisse, die einen Vergleich beider Therapieregime, aufgeschlüsselt nach verschiedenen Patientenkollektiven (z.B. Lungenembolien bei Patienten mit Tumorleiden), ermöglicht. Dies könnte jedoch durchaus klinische Relevanz haben. Darüber hinaus bleiben die Mechanismen einer möglichen Reduktion der Mortalität nach 3 Monaten (4,7 vs. 6,1\%) - wie auch in vorherigen Studien - unklar. In der oben bereits erwähnten Metaanalyse von Gould u. Mitarb. [1] konnte gezeigt werden, dass bei Patienten mit tiefer Beinvenenthrombose die Behandlung mit nieder- molekularem Heparin - im Vergleich zu intravenöse verabreichtem unfraktioniertem Heparin - die Gesamtmortalität signifikant reduziert, dies war jedoch nicht das Resultat einer reduzierten Mortalität infolge einer geringeren Anzahl thromboembolischer Ereignisse oder von weniger Blutungskomplikationen. Letztlich ermöglicht die vorliegende Studie auch keinen sicheren Aufschluss über mögliche Unterschiede hinsichtlich Sicherheit und Effektivität zwischen den verschiedenen niedermolekularen Heparinpräparaten, die sich durchaus hinsichtlich des Molekulargewichtes, der Plasmaclearance und der spezifischer Aktivität unterscheiden [8].

Zusammenfassend legen die Ergebnisse der Metaanalyse von Quinlan u. Mitarb. den Schluss nahe, dass die Therapie mit niedermolekularem Heparin eine sichere und effektive Alternative zu unfraktioniertem Heparin bei der Behandlung von nicht fulminanten Lungenembolien sein kann. Zur abschließenden Beurteilung, ob niedermolekulares, subkutan verabreichtes Heparin routinemäßig in der - auch bevorzugt ambulanten - Behandlung von Patienten mit submassiver Lungenembolie eingesetzt werden kann, bedarf es der Durchführung weiterer Studien mit größeren Patientenzahlen. Neben niedermolekularem Heparin dürften in Zukunft auch selektive Faktor Xa Inhibitoren, von denen sich Fondaparinux in einer Studie aus dem Jahre 2003 als gleichermaßen sicher und effektiv bei der Behandlung von Patienten mit symptomatischer Lungenembolie erwies [9], eine größere Rolle spielen und zur klinischen Anwendung kommen.

\section{Literatur}

${ }^{1}$ Gould MK, Dembitzer AD, Doyle RL et al. Low-molecular-weight heparins compared with unfractionated heparin for treatment of acute deep venous thrombosis. A meta-analysis of randomized, controlled trials. Ann Intern Med 1999; 130: 800-809

${ }^{2}$ Leizorovicz A, Simonneau G, Decousus $\mathrm{H}$ et al. Comparison of efficacy and safety of low molecular weight heparins and unfractionated heparin in initial treatment of deep venous thrombosis: a meta-analysis. BMJ 1994; 309: 299-304

${ }^{3}$ Koopman MM, Prandoni P, Piovella $\mathrm{F}$ et al. Treatment of venous thrombosis with intravenous unfractionated heparin administered in the hospital as compared with subcutaneous low-molecular-weight heparin administered at home. The Tasman Study Group. N Engl J Med 1996; 334: 682-687

${ }^{4}$ Levine K, Gent M, Hirsh J et al. A comparison of low-molecular-weight heparin administered primarily at home with unfractionated heparin administered in the hospital for proximal deep-vein thrombosis. $\mathrm{N}$ Engl J Med 1996; 334: 677-681

${ }^{5}$ Quinlan DJ, McQuillan A, Eikelboom JW. Low-Molecular-Weight Heparin Compared with Intravenous Unfractionated Heparin for Treatment of Pulmonary Embolism. A Meta-Analysis of Randomized, Controlled Trials. Ann Intern Med 2004; 140: 175 - 183

${ }^{6}$ Gould MK, Dembitzer AD, Sanders GD et al. Low-molecular-weight heparins compared with unfractionated heparin for treatment of acute deep venous thrombosis. A Cost-Effectiveness Analysis. Ann Intern Med 1999; 130: 789-799

${ }^{7}$ Warkentin TE, Levine MN, Hirsh J et al. Heparin-induced thrombocytopenia in patients treated with low-molecular-weight heparin or unfractionated heparin. N Engl J Med 1995; 332: 1330-1335

${ }^{8}$ Hirsh J, Levine MN. Low molecular weight heparin. Blood 1992; 79: $1-17$

${ }^{9}$ Buller HR, Davidson BL, Decousus H et al. Matisse Investigators. Subcutaneous fondaparinux versus intravenous unfractionated heparin in the initial treatment of pulmonary embolism. N Engl J Med 2003; 349: $1695-1702$ 